\title{
STACKED BASES FOR MODULES
}

\author{
BY JOEL M. COHEN AND HERMAN GLUCK ${ }^{1}$
}

Communicated by Irving Kaplansky, February 25, 1969

Let $R$ be a principal ideal domain (PID), $B$ a free $R$-module and $A$ a (necessarily free) submodule. Bases $\left\{a_{j}: j \in J\right\}$ and $\left\{b_{i}: i \in I\right\}$ for $A$ and $B$, respectively, are said to be stacked if $J \subset I$ and for each $j \in J$, there is an element $m_{j} \in R$ such that $a_{j}=m_{j} b_{j}$. An obvious necessary condition for the existence of stacked bases is that $B / A$ be a direct sum of cyclic modules.

StACKed bases theOREM. Free modules $A \subset B$ over a PID have stacked bases if and only if $B / A$ is a direct sum of cyclic modules.

The Stacked Bases Theorem was conjectured by Kaplansky in $1954[2, \text { pp. 66, 80 }]^{2}$.

One can interpret this result on the level of chain complexes, as follows. Call a chain complex of $R$-modules elementary if it is isomorphic to one of the form $\cdots 0 \rightarrow m R \subset R \rightarrow 0 \cdots$, where $m \in R$ and all the unlisted terms are 0 . Then we have

Corollary. A chain complex $C$ of modules over a PID is a direct sum of elementary chain complexes if and only if $C$ is free and the homology $H_{*}(C)$ is a direct sum of cyclic modules.

The proof is straightforward. Note that applying the corollary to the chain complex $0 \rightarrow A \subset B \rightarrow 0$ yields the original result.

The Stacked Bases Theorem is well known when $B$ is finitely generated [3, p. 162], but the proof in the general case is quite different. In what follows, let us understand all modules to be over a fixed PID. A presentation $\xi$ of a module $C$ is a short exact sequence

$$
\xi: 0 \rightarrow A \stackrel{\iota}{\rightarrow} B \stackrel{\pi}{\rightarrow} C \rightarrow 0
$$

in which $B$ (and therefore also $A$ ) are free modules. The presentation is said to be stacked if $\iota A$ and $B$ have stacked bases. A subscript attached to $\xi$ indicates that the same subscript should be attached to the other characters. Direct sums of presentations are defined in the obvious way. The following lemmas indicate the division of labor in the proof.

1 The authors were partially supported during the preparation of this paper by grant GP 6895 from the National Science Foundation.

2 Page references are to the 2nd (1969) edition of Kaplansky's book. 
Lemma X. Let $\xi$ be a presentation of the direct sum $C$ of cyclic torsion modules, and let $C^{*}$ be a finitely generated submodule of $C$. Then there is a splitting $\xi=\xi_{1} \oplus \xi_{2}$ such that $C^{*} \subset C_{1}$ and $\xi_{1}$ is finitely generated and hence stacked.

Lemma Y. Again let $\xi$ be a presentation of the direct sum $C$ of cyclic torsion modules, and let $B^{*}$ be a finitely generated submodule of $B$. Then there is a splitting $\xi=\xi_{1} \oplus \xi_{2}$ such that $B^{*} \subset B_{1}$ and $\xi_{1}$ is finitely generated and hence stacked.

Lemma $Z$. Any presentation $\xi$ of a direct sum of cyclic modules is itself a direct sum of countably generated presentations.

The Stacked Bases Theorem follows trivially from these lemmas. For let $\xi$ be a presentation of the direct sum $C$ of cyclic modules. By a simple argument, we may assume that $C$ is a direct sum of cyclic torsion modules. By Lemma $Z$, we may assume that $\xi$ is countably generated. So let $\left\{b_{1}, b_{2}, \cdots\right\}$ be a countable basis for $B$. By Lemma $\mathrm{Y}$, there is a splitting $\xi=\xi_{1} \oplus \xi_{2}^{\prime}$, such that $b_{1} \in B_{1}$ and such that $\xi_{1}$ is finitely generated and hence stacked. Let $b_{2}^{\prime}$ be the projection of $b_{2}$ into $B_{2}^{\prime}$. Then again by Lemma $\mathrm{Y}$ there is a splitting $\xi_{2}^{\prime}=\xi_{2} \oplus \xi_{3}^{\prime}$, such that $b_{2}^{\prime} \in B_{2}$ and such that $\xi_{2}$ is finitely generated and hence stacked. Thus far we have $\xi=\xi_{1} \oplus \xi_{2} \oplus \xi_{3}^{\prime}$, with both $b_{1}$ and $b_{2}$ in $B_{1} \oplus B_{2}$. Continuing in this way, we get a splitting $\xi=\bigoplus_{i=1}^{\infty} \xi_{i}$, with each $\xi_{i}$ finitely generated and hence stacked. Thus $\xi$ itself is stacked.

Details will appear in [1].

\section{BIBLIOGRAPHY}

1. J. M. Cohen and H. Gluck, Stacked bases for modules over principal ideal domains, J. Algebra (to appear).

2. I. Kaplansky, Infinite abelian groups, 2 nd ed., Univ. of Michigan Press, Ann Arbor, Mich., 1969.

3. C. H. Sah, Abstract algebra, Academic Press, New York, 1967.

University of Pennsylvania, Philadelphia, Pennsylvania 19104 